\title{
Functional Genomics, Metabolic Engineering and Mutagenesis Study of Lactic Acid Bacterial Strains in Traditional Food Fermentation, Human Health and Their Potential Applications
}

\author{
Mulatu Workie \\ Ethiopian Institute of Agricultural Research, National Agricultural Biotechnology Research Centre, \\ P.O. Box 2003, Addis Ababa, Ethiopia
}

\begin{abstract}
In the past decades, functional genomics, metabolic engineering and mutagenesis screening have been reported on lactic acid bacteria found in food fermentation. The identification and adaptation of novel processes are being researched and applied into the artisanal and industrial fermentation of foods. Genomics and metabolic engineering have been identified and these innovative processes have been shown to optimize the production of metabolites and enhance the functionality of fermented foods production and processing. In the long history, lactic acid bacteria have been used as a probiotic as well as starter functioning for various traditional food fermentation and human health. Novel probiotic microbial starter cultures are being formulated having diverse health benefits and being used for the production of fermented foods and beverages, functional foods and nutraceuticals and single cell protein. In the last decade, functional genomics approaches have been reported for lactic acid bacteria found in diary fermentation products. However, functional and comparative genomic studies have targeted a variety of foods where much attention has been focused on starter lactic acid bacteria and nonstarter lactic acid bacterial microbial strains. This review is focusing on the potential of functional genomic, metabolic engineering and mutagenesis approaches to unravel the molecular processes in lactic acid bacteria used in industrial fermentation process that determine their functional efficacy in food industry.
\end{abstract}

Keywords: Fermentation, Genomics, Lactic acid bacteria, Metabolic engineering, Mutagenesis

DOI: $10.7176 / \mathrm{ALST} / 83-03$

Publication date: November $30^{\text {th }} 2020$

\section{Background}

Lactic acid bacteria (LAB) are gram-positive and microaerophilic organisms able to ferment sugar to produce lactic acid as an end product and functionally classifications are comprised of the genera Lactobacillus, Lactococcus, Streptococcus, Enterococcus, Leuconostoc and Carnobacterium species. Lactic acid bacteria are considered as safe and used as probiotics functioning aspects of humans. Moreover, when describing their characteristics, LAB is often claimed to have a 'generally recognized as safe' (GRAS) status, which confirms their safety (Stiles and Holzapfel, 1997). Lactic acid bacteria are greatly differing with their morphology, genotypic properties, salt, and acid and temperature tolerance efficiency. Lactic acid bacterial starters reduce fermentation time by overwhelming the fermenting media prior to the colonization of undesirable microbes, in the same token, which reduces production cost and increases quality of the product. In the process of fermentation, they generate lactic acid from the respective sugar fermentation, which makes the environment unconducive for food spoiling and pathogenic microbes, hence, increases the shelf life. The trace and macro biomolecules would increase the nutritional value of the food and the benefit the host acquires up on consumption (Robert, 2006). Lactic acid bacteria are widely exploited for extending the shelf life of various foods and beverages.

Lactic acid bacteria are found everywhere and their abundance is extremely diverse with their specific microbial ecosystems. The selective pressure exerted by these environments is a key driver in the genomic diversity among LAB strains derived from distinct habitats (McAuliffe, 2018).

Naturally Lactic acid bacteria are used as starter culture for various traditional fermented foods like sauerkraut, pickles, cheese, sausage, fish, sourdough bread, and animal silage, fruits, cereals, milk and animal farm environment (Lortal et al., 2014). A plant-based origin for dairy lactococci was demonstrated using genome evolution studies. Starters and ferments have then been isolated, selected, and domesticated over past centuries to control the fermentation processes and standardize the taste and quality of the final products. The total microbial load or count of Lactic acid bacteria in fermented products is highly diverse and ranges from 4 to $5 \log$ to over $9 \log$ of bacteria, depending on product types, fermentation dynamics, and the overall microbial ecology of the products. Moreover, the abundance of other non-starter Lactic acid bacteria, yeasts, and molds in complex ecosystems, especially in dairy products needs to be considered. The microbial diversity and functional properties of lactic acid bacteria found in traditional food products and contribution of Lactic acid bacteria has been extensively reviewed (Tamang et al., 2016; Linares et al., 2017; Marco et al., 2017). Traditional dairy products are one of the major sources of lactic acid bacteria with various health benefits such as prevent from 
various specific diseases.

\section{Functional and comparative genomics of lactic acid bacteria}

In industrial fermentation lactic acid bacterial are widely used for production of dairy products such as cheese and yoghurt are the most commonly produced products. The industry earns multi-billion dollar and great attention has been given to the functional genomics of lactic acid bacteria during fermentation of dairy products. The most commonly used lactic acid bacteria are Lactococcus lactis, Streptococcus thermophilus, Lactobacillus delbruekii subsp. bulgaricus and some Leuconostoc (De Vos, 2011). According to Siezen et al., (2010) the genome sequences of industrial microbial starter strains have not been determined yet or not been made available in public databases.

The recent development of high throughput functional genomics screening approaches such as transcriptomics and metabolomics. Transcriptomics have been used for the analysis of the lactic acid bacteria response to different environments concerning growth conditions, culturing regiments and various stresses and $\mathrm{A}$ lot of studies on the functional genomics contributed to the discovery and characteristics of respiratory metabolism in many bacterial species belonging to lactic acid bacteria (Lechardeur et al., 2011; Pedersen et al., 2012). The proteomic approaches, used to better understand the metabolism of microbial strains in food and the proteomic approach was applied for quantitative analysis of bacterial enzymes released in cheese during ripening and proteomics approach was used to investigate the temperature and acidic stress tolerance response of lactic acid bacterial strains (Nezhad, 2010). The development and use of comparative genomics comparative genomics approaches to improve the functional annotation of the key enzymes in the formation of flavor compounds from amino acids. Also, an integrated database and bioinformatics platform called open genome resource and functional comparative genomics study of probiotic lactic acid bacterial strains. Comparative analysis of the various sequenced lactic acid bacteria resulted in an overview of differences in their capacities to form flavors (Liu et al., 2008). Comparative genomics revealed some parallels between the different phylogenetic strains, which probably reflect the harsh and competitive environment in the human gut.

The genome sequences of twenty lactic acid bacteria have been published to date and the comparative and functional genomic analyses have been performed for some of the species of lactic acid bacteria and their roles in food industry and health have been widely studied (Altermann, 2005). Recently, genomic interest has developed for the nonstarter lactic acid bacterial strains that are naturally present in dairy fermentations products and in some cases have been developed into adjunct starters that contribute to flavor and quality improvement of traditional fermented foods products (Settanni and Moschetti, 2010; Papanikolaou, et al., 2012). The genome size of lactic acid bacteria is relatively small and collectively of low GC content and LAB species with the smallest genomes can be highly auxotrophic and deficient in a number of biosynthetic pathways, corresponding to their apparent adaptation to nutritionally rich environments (Pridmore, Berger et al., 2004; Altermann, Russell et al., 2005).

Moreover, the genomic characterization of Lactobacillus helveticus strain CNRZ 32, used as an adjunct starter to improve the quality and flavor of dairy products and reduce bitterness comparing with other Lactobacilli (Broadbent et al., 2013). Differential Blast Analysis highlighted strain-specific genes and groupspecific genes. Lactobacillus-specific genes include mucus binding proteins involved in cell-adhesion and several transport systems for carbohydrates and amino acids (Klaenhammer et al., 2008). The available genomescale models can play a role as powerful tool as part of comparative genomics. The ultimate goal of the comparative genomics will be a better understanding of the metabolism of these important industrial food starters and why they are so well equipped for their respective food fermentations.

According to Klijn et al., (1995), the genomic based molecular identification of lactic acid bacteria revealed that the fraction of lactic acid bacteria in the human gastro intestinal tract is low and reaches over $1 \%$ in few persons and this indicates many lactic acid bacteria are passengers of the gastro intestinal tract than endogenous inhabitants. The detailed genomic identification of lactic acid bacterial strains is needed to clarify their roles with in the human gastro intestinal tract and some lactic acid bacterial strains have a highly intraspecies diversity in both endogenous and passenger microbial strains. However, genomic fat feeding trail study showed that Lactococcus lactis, Streptococcus thermophilus and Pediococcus acidilacti are commonly used as starter functioning of diary fermentation and meat processing (David et al., 2014).

The genome sequences of eight commensal and fermentative lactic acid bacterial strains have been published: Lactococcus lactis, Lactobacillus plantarum, Lactobacillus johnsonii, Lactobacillus acidophilus, Lactobacillus sakei, Lactobacillus bulgaricus, Lactobacillus salivarius and Streptococcus thermophilus (Altermann and Russell et al., 2005). This indicates the complete genome sequence of other lactic acid bacteria representing the functional diversity of hexose sugar fermenting and lactic acid-producing microbial strains. The lactic acid bacteria have small genomes encoding a range of biosynthetic capabilities that reflect both prototrophic and auxotrophic characters. Phylogenetic and comparative genomic analyses content across the group and reconstruction of ancestral gene sets reveal a combination of gene loss and gain during the 
coevolution of lactic acid bacteria with animals and the foods they consumed. The genome of Lactococcus latics spp. lactis IL1403, released in 2001, represents the first reported lactic acid bacteria genome sequence. Since then, more than 75 genome of industrially important LAB have been sequenced, while more than 80 genome sequencing projects are in progress and GC content of lactic acid bacterial strains are low (Bron and Kleerebezem, 2011).

Comparative genomics and whole genome sequencing provide insights into genetic content, differences and similarities, and offer important clues into possible gene functions, both essential and unique. Genomic analyses of lactic acid bacteria have showed a number of interesting features that are generally considered to be important to the roles of these organisms in food processing industry and health. Adherence and attachment factors and considered potentially important to probiotic functions of lactic acid bacterial strains (Schell et al., 2002). Functional genomic analysis is vital to our understanding of cellular physiology, metabolic pathways, sensing, signaling, and elucidating mechanisms that underlay probiotic functioning. Genetic and molecular tools that can be used for gene cloning, expression, complementation and inactivation.

Recently, functional and comparative genomics has greatly enhanced for the use of a variety of industrial applications. Various starter and probiotic functioning lactic acid bacterial strain Producer Company have been started to characterize and identify their strains using complete genomic analysis. Applying rapid microbial strain identification is useful in mining and selecting the specific properties of lactic acid bacterial strains and their safety and administrative aspects are supported by genome sequencing technology. For comparative genomic study, application and use of high through put sequencing technology allowed for well-known commercial microbial strains.

In the last decade, functional genomics approaches have been reported for lactic acid bacteria found in diary fermentation products. However, functional and comparative genomic studies have targeted a variety of foods where much attention has been focused on starter lactic acid bacteria and non-starter lactic acid bacterial microbial strains.

\section{Mutagenesis study and metabolic engineering of Lactic acid bacteria}

Mutagenesis either physical or chemical can be used to obtain mutant Lactic acid bacteria with the desired functions in biotechnological applications. Apart from screening and isolation of potent lactic acid bacteria from the natural environment, strain improvement of the existing ones also guaranties elite isolates. Simpler but effective strain improvement techniques, like chemical and radiation mutagenesis have been proved to generate improved isolates with vigor potential compared to their wild type isolates. Shorter doubling time, which would reduce the fermentation time, stress tolerance to environmental stress, assimilation of inexpensive and complex raw materials efficiently, overproduction of primary and secondary metabolites are some of the features of improved strains. Random mutagenesis could be adopted in order to enhance food productivity in the dairy sector, where dairy fermentation is characterized by poor infrastructure and uncontrolled fermentation environment (Robert, 2006).

Ethyl methane sulfonate mutagenesis of Lactic acid bacterial has produced variants with improved riboflavin production (Chen et al., 2017). The chemical mutagen (ethyl methane sulfonate) has been mostly applied for random mutagenesis. Lactobacillus subjected to mutagenesis by ethyl methane sulfonate can withstand higher xylose levels and produce larger amounts of lactic acid through conversion of xylose into lactic acid (Almalki, 2016).

There are several drawbacks of random mutagenesis methods. They are non-targeted and can introduce various random mutations into the genomes of interest, after which the characterization and selection of the subset of target variants is required. However, undesired mutations can occur that require further characterization of the isolated strains and on the other hand recombinant DNA technology is targeted and enables precise modifications to be made (Derkx et al., 2014). Moreover, random mutagenesis have been widely applied to obtain improved lactic acid bacteria strains for food applications as strains resulting from these methods are not considered GMO. However, such methods do not result in targeted modifications and selection of the right strains is often laborious, despite bioinformatics tools being highly instrumental to narrow down the initial experimental strain selection. The development of high-throughput genome editing tools for a wide variety of strains is crucial for both fundamental studies and applications to enable fast, targeted and stable genomic modifications (Bergsveinson et al., 2017).

A lot of metabolic engineering techniques have been used to reroute metabolic pathways for the production of sweeteners, flavors, aromas, exopolysaccharides and vitamins (Hugenholtz et al., 2002). Improving the efficiency of lactic acid bacterial strains metabolic engineering is essential. However, more aspects of metabolism cannot be altered by manipulating the desired genes due to the complex network of regulatory mechanisms of cell. In the post-genomic era, metabolic engineering has broadened its design strategies through integrating various biological data, including genomic-scale metabolic model, transcriptomic and metabolomic data (Tomar and De, 2013). In many countries, the use of genetically modified organisms (GMOs) in food 
products is controversial and not allowed by regulatory officials. Development of high-throughput genome editing tools for lactic acid bacteria has been limited compared to industrial strains like Saccharomyces and Escherichia coli. However, lactic acid bacteria are widely studied group for development of genetic tools with many cloning vectors derived from them still routinely used (De Vos, 2011).

Genetically modified lactic acid bacteria can produce significant metabolites with high yield and immense health benefits through metabolic engineering (Liu et al., 2019; Papagianni, 2012). Genetically engineered lactic acid bacterial strain can produce desired compounds and reduces the possibility of the risk of contamination and unwanted release of microbes into the environment. Engineered Lactic acid bacteria have enabled their application to the expression of recombinant proteins and the most common genera are Lactococcus and Lactobacillus (Mao and Wang, 2016; Song et al., 2017). Metabolic engineering of Lactic acid bacteria allows modification of the existing metabolic pathways to improve its properties of Lactic acid bacterial strains as starter functioning of various food fermentation (Berlec and Strukelj, 2009).

\section{Roles of lactic acid bacteria for food fermentation}

In food fermentation industry lactic acid bacteria are one of the ancient known food preserving techniques. Fermented traditional dairy products, such as yogurt and cheese, appeared in human diet about 8,000-10,000 years ago. In the 20th century, food fermentation remained an uncontrolled process; the discovery and characterization of lactic acid bacteria have changed the views on food fermentation. Microbial fermentation enhances the shelf-life of food products and influence the sensory properties of a product including the flavor development and Lactic acid bacterial are among the well-known microbial strains commonly used in industrial food fermentation. Fermented foods also have increased levels of nutrients and are sometimes easier to digest than the raw product. Lactic acid bacteria strains produce bacteriocins, of which nisin is probably the best-known member. Combined with the process of acidification and inhibits the growth of food borne pathogens (Leroy and De Vuyst, 2004).

Microbial starters cultures are classified into two such as defined starter culture and mixed-strain cultures. Defined microbial starter cultures are pure cultures with known physiological characteristics and technological properties and consist of 2-6 strains, used in rotation as paired single strains as multiple strains and enable industrial-scale production of high quality products whereas the mixed microbial starter cultures contain unknown numbers of strains of the same species and may contain bacteria from different genera of lactic acid bacterial strains. Microbial inoculation is one of the traditional methods for the manufacture of fermented food products like back slopping. Back slopping method has its own drawbacks and mainly a great fluctuation in the quality of the product, but is still used for some homemade products. The substitution of the back slopping with a selected starter culture was very early realized to be a necessity. However, the production of fermented foods is automated and produced in large quantities with total control of the process, the use of commercial lactic acid bacterial starter cultures is an integral part of a successful production of any fermented product (Bintsis and Athanasoulas, 2015). In the fermented food industry lactic acid bacterial strains able to inhibit the growth of disease causing and food spoilage microorganisms by lowering the $\mathrm{pH}$ of ecological environment and by competing for nutrients (Ghalfi et al., 2010). Lactic acid bacteria also produce a variety of metabolites, metabolic products, enzymes, lytic agents and bacteriocins, which contribute to the safety and enhance the shelf life of fermented food products (O'Shea et al., 2013). Bacteriocins are among the well-known bioactive substances produced by lactic acid bacterial strains with antimicrobial properties.

\section{Lactic acid bacteria as a starter and probiotic microbes}

According to Marco et al., (2006), probiotic are live microorganism could be viable at the time of ingestion to confer a health benefit and a probiotic microbial strain should survive gastro intestinal tract and colonize the host epithelium. A probiotic functioning microbial strains is able to tolerate both to the highly acidic conditions in the stomach, various concentrations of bile salts in the human small intestine, adherence to intestinal mucosal cells would be necessary for colonization and any direct interactions between the probiotic and host cells leading to the competitive exclusion of pathogens and this properties have consequently become important selection criteria for new probiotic functionality. In food processing industry, lactic acid bacteria have been used as starter cultures for food fermentation or as probiotics microbial strains. Even though, the recent advent of genomics and functional genomics and high-throughput technologies in the last decade have allowed for an extensive understanding of the industrial use and metabolic characteristics of lactic acid bacterial strains, which render them the most promising candidates as microbial cell factories in future bio-refineries (Gaspar et al., 2013).

Lactic acid bacteria (LAB) are commonly used for the production of traditional fermented dairy products and vegetables. The strains should be recognized as safe, holding the GRAS (Generally Recognized as Safe) status for the production of different fermented food or pharmaceuticals. They must also have a number of features in the aspects of safety, functional and technological characteristics. Lactic acid bacteria used in probiotic preparations are derived from the human gastrointestinal tract, are accurately identified, with a history 
of safe use and are not the subject of any reports of their potential pathogenicity or antibiotic resistance (Piatek et al., 2012). Based on the carbohydrate metabolism efficiency lactic acid bacteria can be classified as homofermentative including Lactococcus, Enterococcus, Streptococcus and some lactobacilli use the EmbdenMeyerhof pathway in which glucose is completely converted into lactate and heterofermentative lactic acid bacterial strains composed of Leuconostoc, Weissella and some lactobacilli, produce lactate, acetate, CO2 and ethanol using the hexose monophosphate pathway. Some of the homolactic lactic acid bacteria able to use the mixed acid fermentation for growth, resulting in the formation of mainly acetate, formate and ethanol (Ross et al., 2002).

Lactic acid bacteria have attracted a lot of attention as probiotics, which are used as adjuvant or prophylaxis approach against conditions ranging as broad as neuropsychiatric disorders, cancer, irritable bowel syndrome and urinary tract infections (Reid, 2017; Mays and Nair, 2018). They are also used in a range of animal husbandries including chickens, cows, pigs and fish, to enhance productivity and reduce illnesses (Syngai, 2016). The market for probiotics is ever expanding, with a projected worldwide market size of $\$ 46.55$ billion by 2020 (Salvetti and O'Toole, 2017). The complex molecular mechanistic modes of action of both probiotics and LAB-host-pathogen interactions are not yet fully understood. The development of new starter cultures for the production of fermented foods that could meet the changing consumer preferences and expectations for safe products with specific characteristics is studied through the composition of mixed-strain cultures isolated from nature or the genetic engineering of existing isolates. A variety of techniques involving natural selection and evolution are available to enhance the performance of existing strains, including the isolation of mutants with desired properties, adaptive laboratory evolution, genome shuffling, and genome editing. However, for food applications, recombinant DNA technology is strongly limited by regulations and the negative consumer perspective towards genetically modified organisms (Borner et $a l .$, 2018).

Probiotic lactic acid bacterial strains possess unique features that enable them to survive the rigors of the gastro intestinal tract, survive in the presence of acid and bile, and competition from other microorganisms. Sequencing the genomes of L. acidophilus, L. casei, L. johnsonii, L. plantarum and L. salivarius led to the elucidation of many genes that underlie these characteristics. These species are remarkably deficient in their biosynthetic capacities, which are compensated for by their abundant proteolytic systems and extensive capacity for uptake of macromolecules (Kleerebezem, 2003). Some species of probiotic lactic acid bacteria are able to metabolize complex carbohydrates by human enzymes or by other microbiota and prebiotics can selectively stimulate the growth of lactobacilli in the intestines. Carbohydrates metabolism by L. acidophilus and L. plantarum has identified specific transporters and hydrolases for oligosaccharides (Saulnier, 2007; Barrangou, 2006).

Various in-vitro expermenments could be performed during screening and selection of potential probiotic microbial strains. Among the tests of a probiotic lactic acid bacterial strain, determination of its taxonomic classification is an indication of the origin and physiology of the lactic acid bacterial strains and all the characteristics are important for the selection of the novel probiotic microbial strains (Morelli, 2007). Lactic acid bacteria produce conjugated linoleic acids, gamma-amino butyric acid (GABA) and may contribute to signal the neuroendocrine and vascular systems. More studies are necessary to determine the best strains, optimal dose, and treatment time to achieve beneficial outcomes for obesity, type-2 diabetes, non-alcoholic fatty liver disease (NAFLD) and decipher the corresponding mechanism(s). Collectively, those data should shed light on selected probiotic strains as important tools to prevent and treat patients with metabolic disorders and cardiovascular diseases. Phenotype characterization, genotype stability (plasmid stability; carbohydrate and protein utilization), growth and survival of gastric acid and bile salt tolerance, production of antimicrobial metabolites like bacteriocins able to inhibit pathogens, spoilage microbes, ability to adhere to the intestinal mucosa and antibiotic resistance are one of the major screening and selection criteria for probiotic microbial strains (Tuomola et al., 2001).

\section{Conclusions}

Due to the rapid development of high through put sequencing techniques and multiple genome sequencing projects on lactic acid bacterial strains were initiated and comparative analysis of lactic acid bacteria genomic data emphasized the remarkable diverse within the group at numerous taxonomic levels. Genomics and metabolic engineering have been identified and the processes have been shown to optimize the production of metabolites and enhance the functionality of fermented foods production and processing. Mutagenesis and metabolic engineering of lactic acid bacteria could be adopted in order to enhance food productivity in the dairy sector and human health and to ensure better productive with safer products, saves cost and improves livelihood.

\section{list all abbreviations}

GABA: gamma-amino butyric acid; GRAS: generally recognized as safe'; LAB: Lactic acid bacteria 


\section{Declarations}

The author declare that they have no competing interests

Acknowledgements

Not applicable

Funding

Not applicable

Availability of data and materials

Not applicable

\section{Author contribution}

The author in this manuscript critically review various published papers, develop manuscript and approved the final version of the review manuscript

Ethics approval and consent to participate

Not applicable

Consent for publication

Not applicable

\section{References}

Almalki, M.A. (2016). Production of medically important lactic acid by Lactobacillus Pentosus: A biological conversion method. Indian J. Sci. Technol, 9, 1-8.

Altermann, E., Russell, W., Azcarate-Peril, M., Barrangou, R., Buck, B., McAuliffe, O., Souther, N., Dobson, A., Duong, T., Callanan, M. (2005). Complete genome sequence of the probiotic lactic acid bacterium Lactobacillus acidophilus. Proc Natl Acad Sci, USA 102:3906-3912.

Barrangou, R. (2006). Global analysis of carbohydrate utilization by Lactobacillus acidophilus using cDNA microarrays. Proc. Natl. Acad. Sci. U. S. A. 103, 3816-3821.

Berlec, A. and Strukelj, B. (2009). Novel applications of recombinant lactic acid bacteria in therapy and in metabolic engineering. Recent Pat. Biotechnol, 3, 77-87.

Bergsveinson, J., Kajala, I. and Ziola, B. (2017). Next-generation sequencing approaches for improvement of lactic acid bacteria-fermented plant-based beverages. AIMS Microbiol, 3:8-24.

Bintsis, T. and Athanasoulas, A. (2015). Dairy starter cultures, in: Papademas P, Editor, Dairy Microbiology, A Practical Approach, Boca Raton: CRC Press, 114-154.

Borner, R.A., Kandasamy, V. and Axelsen, A.M. (2018). High-throughput genome editing tools for lactic acid bacteria: Opportunities for food, feed, pharma and biotech. Available from: www.preprints.org.

Bron, P.A and Kleerebezem, M. (2011). Engineering lactic acid bacteria for increased industrial functionality. Bioeng Bugs 2:80-7.

Broadbent, J.R., Hughes, J.E., Welker, D.L., Tompkins, T.A. and Steele, J.L. (2013). Complete genome sequence for Lactobacillus helveticus CNRZ 32, an industrial cheese starter and cheese flavor adjunct. Genome Announc, 1.

Chen, J., Vestergaard, M., Jensen, T.G., Shen, J., Dufva, M., Solem, C. and Jensen, P.R. (2017). Finding the needle in the haystack-the use of microfluidic droplet technology to identify vitamin-secreting lactic acid bacteria, 8, e00526-517.

Derkx, P.M., Janzen, T., Sorensen, K.I., Christensen, J.E., Stuer-Lauridsen, B. and Johansen, E. (2014). The art of strain improvement of industrial lactic acid bacteria without the use of recombinant DNA technology. Microbial Cell Fact, 13, S5.

De Vos W.M. (2011). Systems solutions by lactic acid bacteria: from paradigms to

Practice. Microbial Cell Fact, 10

David, L.A., Maurice, C.F., Carmody, R.N., Gootenberg, D.B., Button, J.E., Wolfe, B.E., Ling, A.V., Devlin, A.S., Varma, Y. and Fischbach, M.A. (2014). Diet rapidly and reproducibly alters the human gut microbiome. Nature, 505:559- 563.

Gaspar, P., Carvalho, A.L. and Vinga, S. (2013). From physiology to systems metabolic engineering for the production of biochemicals by lactic acid bacteria. Biotechnol Adv 31: 764-88.

Ghalfi, H, Benkerroum, N. and Ongena, M. (2010). Production of three anti-listerial peptides by Lactobacillus curvatus in MRS broth. Food Res Int, 43:33-9.

Hugenholtz, J., Sybesma, W., Nierop Groot, M., Wisselink, W., Ladero, V., Burgess, K., van Sinderen, D., Piard, J.C., Eggink, G. and Smid, E.J. (2002). Metabolic engineering of lactic acid bacteria for the production of nutraceuticals. Lactic Acid Bacteria Genet. Metab. Appl, 82, 217-235.

Klaenhammer, T. R., E. Altermann, E. Pfeiler, B. L. Buck, Y. J. Goh, S. O'Flaherty, R. Barrangou, and T. Duong. (2008). Functional genomics of probiotic Lactobacilli. J Clin Gastroenterol, 42, 160-162.

Klijn, N., Weerkamp, A.H. and De Vos, W.M. (1995). Genetic marking of Lactococcus lactis shows its survival in the human gastrointestinal tract. Appl Environ. Microbiol, 61:2771-2774. 
Kleerebezem, M. (2003). Complete genome sequence of Lactobacillus plantarum WCFS1. Proc. Natl. Acad. Sci. U. S. A. 100, 1990-1995

Lechardeur, D., Cesselin, B., Fernandez, A., Lamberet, G., Garrigues, C., Pedersen, M., Gaudu, P. and Gruss, A. (2011). Using heme as an energy boost for lactic acid bacteria. Current Opinion in Biotechnology. 22 (2), pp. 143-149.

Leroy, F., and L. De Vuyst. (2004). Lactic acid bacteria as functional starter cultures for the food fermentation industry. Trends Food Sci Technol, 15:67-78.

Linares, D. M., Gómez, C., Renes, E., Fresno, J. M., Tornadijo, M. E., Ross, R. P. (2017). Lactic acid bacteria and bifidobacteria with potential to design natural biofunctional health-promoting dairy foods. Front. Microbiol. 8:846. doi: 10.3389/fmicb.2017.00846.

Liu, J., Chan, S.H.J., Chen, J., Solem, C. and Jensen, P.R. (2019). Systems Biology-A guide for understanding and developing improved strains of lactic acid bacteria. Front. Microbiol, 10, 876.

Liu, M., A. Nauta, C. Francke, and R. J. Siezen. (2008). Comparative genomics of enzymes in flavor-forming pathways from amino acids in lactic acid bacteria. Appl Environ Microbiol, 74:4590-4600.

Lortal, S., Licitra, G., and Valence, F. (2014). Wooden tools: reservoirs of microbial biodiversity in traditional cheesemaking. Microbiol. Spectr. 2:CM-000- 2012.doi: 10.1128/microbiolspec.CM-0008-2012

Mao, R., Wu, D. and Wang, Y. (2016). Surface display on lactic acid bacteria without genetic modification: Strategies and applications. Appl. Microbiol. Biotechnol, 100, 9407-9421.

Marco, M. L., Pavan, S. and Kleerebezem, M. (2006). Towards understanding molecular modes of probiotic action. Curr Opin Biotechnol. 17:204-210.

Marco, M. L., Heeney, D., Binda, S., Cifelli, C. J., Cotter, P. D., Foligné, B. (2017). Health benefits of fermented foods: microbiota and beyond. Curr. Opin. Biotechnol. 44, 94-102. doi: 10.1016/j.copbio.2016.11.010.

Mays, Z.J. and Nair, N.U. (2018). Synthetic biology in probiotic lactic acid bacteria: At the frontier of living therapeutics. Curr Opin Biotechnol, 53:224-31.

McAuliffe, O. (2018). Symposium review: Lactococcus lactis from nondairy sources: their genetic and metabolic diversity and potential applications in cheese. J. Dairy Sci. 101, 3597-3610. doi: $10.3168 /$ jds.2017-13331

Morelli, L. (2007). In vitro assessment of probiotic bacteria: From survival to functionality. International Dairy Journal, 17, 1278-1283.

Nezhad, M.H. (2010). Effect of growth at low pH on the cell surface properties of a typical strain of $<$ em $>$ Lactobacillus casei</em> group [online]. Iranian journal of microbiology. 2 (3).

O'Shea, E.F., Cotter, P.D., Ross, R.P. and Hill, C. (2013). Strategies to improve the bacteriocin protection provided by lactic acid bacteria. Curr Opin Biotechnol 24:130-4.

Papagianni, M. (2012). Metabolic engineering of lactic acid bacteria for the production of industrially important compounds. Comput. Struct. Biotechnol, 3, e201210003.

Papanikolaou, Z., Hatzikamari, M., Georgakopoulos, P., Yiangou, M., Litopoulou Tzanetaki, E. and Tzanetakis N. (2012). Selection of dominates NSLAB from a mature traditional cheese according to their technological properties and in vitro intestinal challenges. J Food Sci, 77:M298-306.

Pedersen, M.B., Gaudu, P., Lechardeur, D., Petit, M.A. and Gruss, A. (2012). Aerobic respiration metabolism in lactic acid bacteria and uses in biotechnology. Annual Review of Food Science and Technology. 3pp. 37-58.

Piątek, J., Gibas-Dorna, M. and Olejnik A. (2012). The viability and intestinal epithelial cell adhesion of probiotic strain combination -in vitro study. Ann Agric Environ Med, 19: 99-102.

Pridmore, R.D., Berger, B., Desiere, F., Vilanova, D., Barretto, C., Pittet, A.C., Zwahlen, M.C., Rouvet, M., Altermann, E., Barrangou, R., Mollet, B., Mercenier, A., Klaenhammer, T.R., Arigoni, F. and Schell, M.A. (2004) The genome sequence of the probiotic intestinal bacterium Lactobacillus johnsonii NCC 533. Proc. Natl. Acad. Sci. USA 101, 2512-2517.

Reid, G. (2017). The development of probiotics for women's health. Can J Microbiol,63:269-77.

Robert, W.H., 2006. Microbiology and technology of fermented foods. Blackwell Publishing, Ames, Iowa, USA.

Ross, R. P., S. Morgan, and C. Hill. (2002). Preservation and fermentation: past, present and future. Int J Food Microbiol, 79:3-16.

Saulnier, D.M. (2007). Identification of prebiotic fructooligosaccharide metabolism in Lactobacillus plantarum WCFS1 through microarrays. Appl. Environ. Microbiol. 73, 1753-1765.

Salvetti, E. and O'Toole, P.W. (2017). When regulation challenges innovation: The case of the genus Lactobacillus. Trends Food Sci Technol, 66:187-94.

Schell, M.A., Karmirantzou, M., Snel, B., Vilanova, D., Berger, B., Pessi, G., Zwahlen, M.C., Desiere, F., Bork, P., Delley, M., Pridmore, R.D. and Arigoni, F. (2002). The genome sequence of Bifidobacterium longum reflects its adaptation to the human gastrointestinal tract. Proc. Natl. Acad. Sci. USA 99, 14422-14427.

Stiles, M.E. and Holzapfel, W.H. (1997). Lactic acid bacteria of foods and their Current taxonomy. Int. J. Food Microbial, 36, 1-29. 
Song, A.A., In, L.L.A., Lim, S.H.E. and Rahim, R.A. (2017). A review on Lactococcus lactis: From food to factory. Microb. Cell Fact, 16, 55.

Siezen, R.J., Bayjanov, J., Renckens, B., Wels, M., Molenaar, D and van Hylckama Vlieg, J.E.T. (2010). Complete genome sequence of Lactococcus lactis subsp. lactis KF147, a plant-associated lactic acid bacterium. J. Bacteriol, 192:2649- 2650.

Settanni, L. and Moschetti, G. (2010). Non-starter lactic acid bacteria used to improve cheese quality and provide health benefits. Food Microbiol, 27:691- 697.

Syngai, G.G., Gopi, R. and Bharali, R. (2016). Probiotics - the versatile functional food ingredients. J Food Sci Technol, 53:921-33

Tamang, J. P., Shin, D. H., Jung, S. J., and Chae, S. W. (2016). Functional properties of microorganisms in fermented foods. Front. Microbiol. 7:578. doi: 10.3389/fmicb.2016.00578

Tomar, N. and De, R.K. (2013). Comparing methods for metabolic network analysis and an application to metabolic engineering. Gene 521:1-14.

Tuomola, E., R. Crittenden, M. Playne, E. Isolauri, and S. Salminen. (2001). Quality assurance criteria for probiotic bacteria. Am J Clin Nutr, 73:393S -398S. 